\title{
Yeast diversity and species recovery rates from beech forest soils
}

Andrey M. Yurkov ${ }^{1}$, Thorsten Wehde ${ }^{2}$, Julian Federici ${ }^{2}$, Angela M. Schäfer ${ }^{2}$, Malte Ebinghaus ${ }^{2}$, Sascha Lotze-Engelhard ${ }^{2}$, Moritz Mittelbach ${ }^{2}$, René Prior ${ }^{2}$, Christian Richter ${ }^{2,3}$, Oliver Röhl' ${ }^{2}$, Dominik Begerow ${ }^{2}$

1 Leibniz Institute DSMZ-German Collection of Microorganisms and Cell Cultures, Inhoffenstraße 7B, 38124 Braunschweig, Germany

${ }^{2}$ Geobotany, Faculty of Biology and Biotechnology, Ruhr-University Bochum, 44801 Bochum, Germany

${ }^{3}$ Microbial Drugs, Helmholtz Centre for Infection Research, Inhoffenstraße 7, 38124 Braunschweig, Germany

\begin{abstract}
Soil yeasts are globally diverse. They are found in almost all soil types, and the structure of soil yeast communities reflects above ground vegetation properties. Cultivation techniques have often been successfully employed to study yeasts in forest soils. However, only few studies have addressed the variation of soil yeast communities in space and time, especially structural dynamics at a forest site between different seasons is unknown. Here, we analyse the result from our field experiments performed in 2008 and 2009. We reassess species inventory data and identify potential new species. Using yeast improved species lists we estimate the rate of species recovery from beech forest soils with a particular focus on repeated sampling. Our analyses showed that the number of observed yeast species was steadily increasing after one, two and three samplings. The observed diversity was likely approaching saturation after four samplings. Additionally, we provide formal descriptions of new yeast species isolated from forest soils in Germany during these studies, as $30 \%$ of the observed species represented undescribed taxa. The following taxonomic novelties are proposed: Colacogloea demeterae Yurkov, Schäfer \& Begerow sp. nov. (MB 816166), Slooffia velesii Federici, Röhl \& Begerow sp. nov. (MB 816165), Hamamotoa cerberi Yurkov, Schäfer \& Begerow sp. nov. (MB 816164), Hamamotoa telluris Yurkov, Schäfer \& Begerow sp. nov. (MB 816163), Piskurozyma yama Richter, Mittelbach \& Begerow, sp. nov. (MB 816162), Piskurozyma tuonelana Lotze-Engelhard, Richter \& Begerow sp. nov. (MB 816161), Dioszegia dumuzii Ebinghaus, Prior \& Begerow sp. nov. (MB 816160), Chernovia houtui Federici, Yurkov \& Begerow gen. nov. et sp. nov. (MB 816158, MB 816159).
\end{abstract}

Corresponding author: Andrey M. Yurkov < andrey.yurkov@dsmz.de> 


\section{Introduction}

Soil is one of the most complex habitats. It is rich in microorganisms and diverse communities of prokaryotes, protists and fungi were described from several studies (e.g. Fierer et al. 2009; Tedersoo et al. 2014). Due to the heterogeneity of the habitat, distribution of microbial taxa in soils is often highly uneven and spatially aggregated on various scales (e.g. Fierer and Jackson 2006; Tedersoo et al. 2014). While a limited number of species are frequently detected from soils, a vast majority of microorganisms appear at very low frequencies and are referred to as "rare biosphere" (Sogin et al. 2006; Lynch and Neufeld 2015). On a global scale these rare taxa contribute to a nearly limitless pool of genetic diversity. Similar to prokaryotes, the diversity of soil fungi was estimated to be very large with only a small number of species overlapping between sampling sites (e.g. Taylor et al. 2010; Wubet et al. 2012).

Yeasts are common soil inhabitants and have been isolated from nearly every type of soil (e.g. Botha 2011; Connell et al. 2008; Vishniac 2006). In forest soils, soil yeast communities are spatially heterogeneous and reflect properties of the above ground vegetation. The diversity of these communities is influenced by plant species (Maksimova and Chernov 2004; Yurkov et al. 2015), forest management history (Yurkov et al. 2012a) and plant projective cover (Yurkov et al. 2016). Extensive isolation and cultivation with subsequent statistical analysis of the data enabled the estimation of the expected species richness in a forest (e.g. Yurkov et al. 2011; Yurkov et al. 2016). Using this approach, recent studies reported a remarkably high proportion of potentially unknown taxa from forest soils (Yurkov et al. 2012a; Yurkov et al. 2016). Recent studies demonstrated that spatial distances and regional heterogeneity contributed to the total diversity values in forest biotopes on long-distance (Yurkov et al. 2015), regional (Yurkov et al. 2012a) and local (Yurkov et al. 2011; Yurkov et al. 2016) scales. Less is known about the dissimilarity of soil yeast communities at the same forest sites by repeated sampling. A few studies suggested that seasonal changes affect yeast community composition in beech forest soils (e.g. Jensen 1963; Yurkov et al. 2011) or in rhizosphere of two herbaceous plants in a birch forest (Golubtsova et al. 2007). However, neither of the afore-mentioned studies addressed the influence of repeated sampling on total yeast diversity values and the proportion of newly discovered species with every consecutive sampling.

Here, we present the results obtained during previous studies on 'very intensively studied' experimental plots (VIPs) of the Biodiversity Exploratories framework. The studies were performed in two consecutive sampling campaigns in 2008 and 2009, and in the present manuscript we aim to estimate the rate of species recovery from beech forest soils and focusing on repeated sampling. For this purpose, we revise species inventory, identify potential new species and provide formal descriptions of new yeast species.

\section{Materials and Methods}

Soil samples were collected from two different localities in Germany on 'very intensively studied' experimental plots (VIPs) of the German Biodiversity Exploratories framework (www.biodiversity-exploratories.de). Study sites were the Hainich National Park (Nationalpark Hainich and its surroundings) in Thuringia (Central Germany) and the Swabian Alb Biosphere Reserve (Biosphärengebiet Schwäbische Alb) in Baden-Wuerttemberg (South-Western Germany). In each region, surveyed plots contained six beech forests of two management types (near-natural and managed). Detailed characteristics of sampling sites can be found in Fischer et al. (2010) and Yurkov et al. (2012a). 
Soils were sampled in April, June, August and October 2009, and studied using the approach described by Yurkov et al. (2012a). Soil yeasts isolated from samples collected in April-May 2008 were studied by Yurkov et al. (2012a).

Yeasts were isolated by plating soil suspension on solid acidified GPYA medium following the method described by Yurkov et al. (2012a). Isolation on a nitrogen-depleted medium (TMV agar and modified Brown's agar) was performed following the method described by Yurkov et al. (2011). Due to low isolation effort (e.g. Yurkov et al. 2011), only samples collected in 2008 were additionally studied with nitrogen-depleted media.

\section{Physiological and biochemical character- istics}

Mating experiments were performed using Potato-Dextrose (= PDA), Corn Meal (= CMA), Yeast Mold $(=\mathrm{YM})$ and Malt-extract, Yeastextract, Soytone (= MYP) agars (Kurtzman et al. 2011; Sampaio et al. 2003; Yarrow 1998). Phenotypic characterisation of isolates was carried out according to Yarrow (1998) and Kurtzman et al. (2011) using both solid and liquid media. Additional assimilation tests using aldaric acids and aromatic compounds were performed as described by Fonseca (1992) and Sampaio (1999), respectively.

\section{Molecular characterisation}

Detailed protocols describing DNA extraction, amplification, purification and sequencing are given by Yurkov et al. (2012a). In short, PCRfingerprinting with minisatellite-specific oligonucleotides derived from the core sequence of bacteriophage M13 with the sequence published by Sampaio et al. (2001) or microsatellite-specific oligonucleotides $(\mathrm{GTG})_{5}$, as a single PCR primer (Gadanho and Sampaio 2002) were used to group pure cultures. Strains from the same sampling event and same sampling site showing identical electrophoretic profiles were considered as con- specific and only 1-2 representatives were chosen for further identification by sequencing of rRNA gene regions.

Yeast cultures were identified using nucleotide sequences of the D1/D2 domains of the large subunit (26S/28S or LSU) rRNA gene. The internal transcribed spacer (ITS) region of the rRNA locus was also sequenced in few cases. Sequencing of the ribosomal small subunit (18S or SSU) was performed as described before (Glushakova et al. 2010). The assembly and editing of sequence data were performed using Sequencher 5.3 (Gene Codes Corp., USA). The nucleotide sequences were compared with sequences deposited in the NCBI (www.ncbi.nih.gov) and CBS (www.cbs.knaw.nl) databases, respectively. Nucleotide sequences were deposited in NCBI/EMBL GenBank under the accession numbers, which are provided in the text.

Pairwise sequence similarity and coverage were used to determine potentially novel species. Thereafter, sequence divergence in the respective phylogenetic genus or clade was considered and a phylogenetic analysis was performed. Sequences were aligned with the MAFFT algorithm, version 7 (Katoh and Standley 2013). Maximum likelihood analysis was performed with RaxML (version 7.4.2) using raxmIGUI 1.3.1 (Silvestro and Michalak 2012) and the GTRCAT option with 100 or 1,000 rounds of bootstrap replicates (Stamatakis et al. 2008).

\section{Statistical data analyses}

Each plot was represented by 4 sub-samples in 4-6 physical replicates (different soil-towater ratios) and 2-3 plates each. Out of 192 sub-samples collected in 2009, a total of 181 were included in the analysis and 11 subsamples were excluded from the analysis because they yielded no yeast cultures either due to low fungal quantity in a particular replicate or due to fast development of moulds, which made isolation and appropriate quanti- 
fication of yeasts difficult. Species inventories recorded for each sub-sample were combined to produce a species list for each plot (a total of six) at each sampling time (a total of four). Thus, the data derived from 181 physical sub-samples was used to produce a total of 24 species inventories.

Because of the limited amount of data (24 species inventories), statistical analyses were limited to the following procedures. Species inventories obtained for each plot of the same management intensity (managed and nearnatural: three plots each) and location (two locations: Swabian Alb and Hainich), and sampling time (April, June, August, October). These values were randomly recombined to produce a table containing the information regarding the number of species isolated after a single sampling, any two samplings, any three samplings and after the continuous sampling during the entire vegetative season (four samplings). This step has been repeated for shared species and species found after every additional sampling without any regard to the region of sampling or forest management type. For each sampling we calculated first, second (median) and third quartiles. Species discovery rates in relation to the number of samplings (one to four) were plotted presented as box plots.

\section{Results and discussion}

A total of 760 yeast cultures were isolated and identified from sub-samples collected in 2009. A total of 35 yeast species (25 basidiomycetes and 10 ascomycetes) were identified (Table 1). Yeasts in soils collected in April-May 2008 were studied and reported by Yurkov et al. (2012a). Here, we describe the species recovered exclusively on nitrogendepleted media as well as two single strains isolated during the study by Yurkov et al. (2012a). The reassessment of yeasts isolated from soils collected in April-May 2008 added two more species isolated on nutrient-rich media and 13 species recovered on nitrogenfree media (Table 1). Taken together, this makes our study the most comprehensive inventory of soil yeasts from beech forest soils.

Among samples collected in 2009, a total of 11 species were shared between Swabian Alb (southern Germany) and Hainich (central Germany). A total of 14 and 9 species were restricted to Swabian Alb and Hainich, respectively. The average species richness ranged between 2 and 3 species per subsample. The number of species isolated during a single sampling varied between 5 and 13 species, representing a single combination of region, vegetation type, land management category and season (Table 2).

Our analyses showed that the number of observed yeast species was steadily increasing after one, two and three samplings. The observed diversity was likely approaching saturation after four samplings (Figure 1). Species inventories from samplings performed in spring 2008 (see Yurkov et al. 2012a) and 2009 also differed substantially with approximately 5 more species to be additionally expected from the sampling in the second year (Figure 2; Table 2). These results show that repeated sampling may substantially improve species inventories of yeasts in soils underneath beech forests.

Studies performed in beech forests on intensively studied experimental plots (VIPs) of the German Biodiversity Exploratories framework yielded a substantial number of novel species (e.g. Yurkov et al. 2012a), some of which have been described previously (e.g. Yurkov et al. 2009a, 2009b; Yurkov et al. 2012b). Comparing the species inventory of this study to previous studies (Yurkov et al. 2011; Yurkov et al. 2012a, 2012c), 20 more species were recovered from forest soils, namely Apiotrichum laibachii, Arthroascus schoenii, Barnettozyma vustinii, Candida chilensis, C. cretensis, C. vartiovaarae, C. zeylanoides, 
Candida sp. TSN-36, Cystofilobasidium macerans, Curvibasidium cygneicollum, Debaryomyces hansenii, Holtermanniella wattica, Phaffia sp. TSN-67, Sporobolomyces ruberrimus, Schizoblastosporion starkeyi-henricii, Slooffia tsugae, Vanrija albida, V. musci, Vishniacozyma tephrensis, and Wickerhamomyces anomalus. Thus, out of 56 species reported to date from the aforementioned forests, approximately $32 \%$ (18 species) represented new species at the date of discovery. Several studies that aimed to estimate the total diversity of fungi suggested the major portion of fungal diversity is unknown to science (Hawksworth 2001; Blackwell 2011). Despite endemism of soil fungi (e.g. Tedersoo et al. 2014), the high proportion of unknown yeast species (reaching $30 \%$ ), which was isolated from a commonly studied niche, such as soil, right in front the doorstep was not expected.

Among ascomycetes, yeasts matching Candida santamariae were isolated from April to August from both regions. Nucleotide sequences of both ITS rRNA and LSU rRNA of these strains deviated from the ones of type strains of the two varieties suggesting that they may represent a complex of cryptic species. The first haplotype represented by the strain TSN-198 (= MUCL 54083, GenBank LT548269) showed four and two substitutions in LSU rRNA to C. santamariae var. santamariae and C. santamariae var. membranifaciens, respectively. The second haplotype represented by the strain TSN-107 (= MUCL 54082, GenBank LT548268) showed five and three substitutions in LSU rRNA to $C$. santamariae var. santamariae and C. santamariae var. membranifaciens, respectively. Similarly, TSN-198 differed in 2 and 3 from the sequences of $C$. santamariae var. santamariae and C. santamariae var. membranifaciens, respectively. ITS sequences of TSN-107 showed 1 and 2 substitutions to $C$. santamariae var. santamariae and $C$. santamariae var. membranifaciens, respectively. The strain TSN-198 differed in 1 nucleotide position (indel) from Candida santamariae strain DBVPG 5182 (GenBank GQ911489), which has been isolated from the Calderone Glacier (Branda et al. 2010).

Sequences of LSU rRNA of two ascomycetous yeast strains TSN-747 and TSN-806 showed low similarity (below 95\%) to hitherto described species. Phylogenetic analyses placed this species in the Candida tanzawaensis clade in the subclass comprised by C. prunicola, C. xylopsoci, C. pyralidae and $C$. vadensis (Fig. 3 ). This is the second new species from this clade, which has been isolated from a forest soil. Strain TSN-36 (= CBS 11767) conspecific to Candida sp. CBS 7170 has been obtained from soils collected in Swabian Alb (Yurkov et al. 2012c). Description of the two novel species should be done in the future together with the complete reclassification of the entire Candida tanzawaensis clade, which is beyond the scope of this study.

Strain TSN-505 showed moderate similarity to species of the genus Saccharomyces. Phylogenetic analyses using combined analysis of SSU and LSU rRNA genes showed its basal position to the genus Saccharomyces and outside the genus Kazachstania, even though the support was very low (data not shown). Again, a formal description needs additional data, which would go beyond the scope of this study.

Following the "one fungus = one name" principle, systematics of basidiomycetous yeasts was recently revised and unified with the classification of filamentous (often sexual) fungi (Liu et al. 2015; Wang et al. 2015). Also, the polyphyletic genera Cryptococcus and Rhodotorula were restricted to the lineages, where the type species belong. Phylogenetic analyses performed in these studies also included nucleotide sequences of yeasts, which were isolated during our surveys. The placement of some yeasts, however, re- 
mained doubtful. Specifically, strain TSN-574 showed little similarity to hitherto described species or known isolates. It was loosely connected to Pseudoleucosporidium fasciculatum and to an undescribed yeast species Rhodotorula sp. CBS 10235 (GenBank EU002852) (data not shown). Proper description of this species will require a multigene analysis as suggested by Wang et al. (2015), which will be performed in a separate study.

Recently mitosporic Microbotryomycetes previously classified in the genera Bensingtonia, Rhodotorula and Sporobolomyces were studied using multi-gene phylogenetic analyses and a constrained analysis of LSU rRNA (Wang et al. 2015). Despite the advantage of the seven-gene backbone tree, clades in the LSU rRNA-based tree often displayed lower support values. Taking into consideration the results of phylogenetic relationships in Microbotryomycetes in previous studies, the authors confirmed a limited utility of LSU rRNA as a phylogenetic marker for this group of yeasts. In our study, several potentially new species from the class Microbotryomycetes were recovered from soils using nitrogen-depleted media. Two novel species represented by strains HEW-1-21 (Rhodotorula sp. AY211 strain CBS 11585, GenBank FN428972) and HEW-1-23 (Rhodotorula sp. AY207 strain CBS 11713, GenBank FN428971) isolated from forest soils in Hainich National Park clustered together with Hamamotoa (Sporobolomyces) singularis (GenBank AF189996) but with low statistical support in the ML analysis (Fig. 4). Although bootstrap support value for this clade was low, the two strains showed $99 \%$ similarity and 8 and 9 nucleotide substitutions in LSU rRNA to $H$. singularis (GenBank AF189996), respectively. Pairwise similarities between strains HEW-1-21 and HEW-1-23 were 99\% (3 substitutions) for LSU rRNA but only $92 \%$ for ITS rRNA. Thus, they are likely to belong to two different species. Based on high sequence similarity values and considering generally low statistical support in LSU-based phylogenetic analyses, we propose these two yeasts as members of the genus Hamamotoa.

Our results were in agreement with the phylogenetic analysis performed by Wang et al. (2015) regarding the placement of a new species (Rhodotorula sp. AY165 strain CBS 12500, GenBank FN428968) in the genus Colacogloea (Fig. 4). This species was represented by two strains AEG-8-20, AEG-9-20 isolated from grassland soils in Swabian Alb. Another new species (Rhodotorula sp. AY167 strain CBS 11664, GenBank FN428962) represented by three strains HEG-1-20, HEG-320 and AEG-8-21 (grassland soils, Hainich National Park and Swabian Alb) was placed in the genus Slooffia (Fig. 4). Below we provide formal descriptions of these species.

Cultivation experiments using both nutrientrich and nitrogen depleted media yielded two isolates AEG-2-20 and HEW-2-3, which were isolated from grassland soil collected in Swabian Alb and from forest soil collected in Hainich National Park, respectively. The two strains AEG-2-20 (= CBS 11560, GenBank FN428969) and HEW-2-3 (= CBS 11579, GenBank FN428970) showed identical LSU and ITS sequences suggesting their conspecificity. They clustered close to Fellozyma inositophila ( $97 \%$ similarity, 15 substitutions) but received no support in the analysis (Fig. 4 ). Because neither sequence similarity values nor statistical support in the phylogenetic analysis are convincing enough to place this species in the genus Fellozyma, proper description of this species will require a multigene analysis as suggested by Wang et al. (2015), and will be performed in a separate study.

In Tremellomycetes, four novel species were discovered. Two novel species represented by strains TSN-538 and TSN-649 (forest soils, Hainich National Park) were placed in the genus Piskurozyma in the phylogenetic 
analysis performed by Liu et al. (2015) and in the present study (Fig. 5). They were closely related to Piskurozyma (Cryptococcus) fildensis and showed 99\% (7 substitutions) and $98 \%$ (12 substitutions) similarity in LSU rRNA, respectively. Pairwise similarity results between strains TSN-538 and TSN-649 were only $95 \%$ for the ITS-LSU rRNA fragment. Thus, they are likely to belong to two different species (Fig. 5). Another new species represented by two strains TSN-530 and TSN-531 (forest soils, Swabian Alb) was placed in the genus Dioszegia (Fig. 6) close to D. cryoxeri$c a$, which has been isolated from soil in Antarctica (Connell et al. 2010). The novel species showed $98 \%$ similarity (11 substitutions) in ITS rRNA and 99\% (4 substitutions) in the LSU rRNA locus, respectively. Below we provide formal descriptions of these species.

Cultivation experiments with forest soils collected Hainich National Park and nitrogendepleted media yielded a yeast strain AY552 (= CBS 12229, GenBank FR820582), which was distantly related $(90-93 \%$ similarity in LSU rRNA) to members of the Cryptococcus victoriae clade. Recent phylogenetic analysis performed by Liu et al. (2015) demonstrated that it represents a hitherto undescribed species in the newly introduced genus Vishniacozyma. Again, a formal description needs additional data, which would go beyond the scope of this study.

Surprisingly, our cultivation experiments yielded two psychrophilic yeasts, Mrakia geliga and $M$. frigida, which are rare outside cold habitats such as glaciers and cryogenic soils (Fell 2011). Although, these yeasts do not grow well even at moderate temperatures, we were able to culture them from temperate forest soils collected in August and October, respectively (Table 1; Figure $\mathrm{S} 1$ ). Although, isolation of $M$. frigida from boreal forest soils has been reported before (Maksimova and Chernov 2004), the reliability of the identification in that study has been questioned later by Fell (2011) since this species does not grow above $20^{\circ} \mathrm{C}$ (Fell 2011). Because LSU rRNA analysis does not have sufficient specificity to differentiate $M$. frigida and $M$. gelida, we identified members of the genus Mrakia using ITS rRNA sequences, as suggested by Fell (2011). The phylogenetic analysis confirmed the identification of our isolate as $M$. frigida (Figure S1). Thus, the present study provides the first report on isolation of $M$. frigida from temperate forest soils. However, it is important to mention that the ITS rRNA sequence of the strain $M$. frigida TSN-231 differed in 7 nucleotide positions (99\% similarity) from sequences of the type strain CBS 5270 (GenBank AF144483).

Among cultures isolated on a nitrogendepleted medium, strain AY409 (forest soil, Schorfheide-Chorin Biosphere Reserve in Brandenburg) was preliminary identified as Cryptococcus sp. from Cystofilobasidiales. However, LSU rRNA and SSU rRNA sequences showed low similarity to Cystofilobasidiales (data not shown) but rather to the genus Basidioascus (Nguyen et al. 2013). Analysis of the concatenated dataset SSU rRNA and LSU rRNA dataset confirmed the placement of this strain in Wallemiomycetes (Fig. 7). This is the first exclusively unicellular growing organism in this class. Members of the genus Basidioascus can produce arthroconidia but typical budding yeast appearance has been observed in $B$. persicus only (Nasr et al. 2014). Formation of hyphal structures, ballistoconidia or sexual reproduction was not observed in the yeast AY409. Taking into consideration its phylogenetic position, distant to the genus Basidioascus, we describe a novel genus to accommodate this yeast.

\section{Taxonomy}

Descriptions of several species provided in this study were made on a single isolate. Although a minimal number of isolates sufficient for a valid taxonomic description is not specified formally, we agree that some morpholog- 
ical and physiological characters can be corrected in the future with the discovery of additional isolates. It is important to document that since the isolation in 2008-2010 and despite several attempts no additional strains have been obtained. In our opinion these yeasts represent rare soil species, and their ecology and distribution patterns cannot be resolved within a single study. Phylogenetic analyses also showed that these yeasts belong to novel or presently undersampled phylogenetic clades. We believe that every new described species is enlarging our knowledge of soil yeasts that may result in a solid hypothesis concerning the ecology of rare species in the future. Thus, we think it is important to describe these species formally in this study.

Since the species have been isolated from soils, they are all named after chthonic gods and goddesses, and deities of the earth.

\section{Description of Colacogloea demeterae} Yurkov, Schäfer \& Begerow, sp. nov. (MB 816166)

Etymology: The specific epithet "demeteriae" derives from the Greek mythological figure of Demeter, a goddess of the harvest and soil fertility.

After growth on YM agar plates for 1 mo at $16^{\circ} \mathrm{C}$ and $22^{\circ} \mathrm{C}$, the streak culture is white to off-white, glistening, mucoid and smooth. The margin is entire. After growth on $\mathrm{YM}$ agar plates for $7 \mathrm{~d}$ at $16{ }^{\circ} \mathrm{C}$ and $22^{\circ} \mathrm{C}$, cells are ovoid to ellipsoid $(3-4 \times 6-8 \mu \mathrm{m})$, occurring singly or in pairs, and proliferating by polar budding. Pseudohyphae and true hyphae were not observed after 1 mo in Dalmau plate culture on corn meal agar at $16-22{ }^{\circ} \mathrm{C}$.

Assimilation of carbon compounds: Growth on D-glucose, D-galactose, D-xylose, Larabinose, D-arabinose, sucrose, a, atrehalose, raffinose, melezitose, D-glucitol, D- mannitol, myo-inositol, D-glucoronate, DLlactate, succinate, citrate. Weak growth on glycerol and ribitol. No growth on L-sorbose, D-glucosamine, D-ribose (or weak), Lrhamnose, maltose (or weak), cellobiose (or weak), lactose (or weak), inulin, starch and ethanol. Assimilation of nitrogen compounds: growth on potassium nitrate, sodium nitrite, Llysine, D-tryptophan and imidazol. Maximum growth temperature: $25^{\circ} \mathrm{C}$.

Molecular characteristics (type strain): nucleotide sequences of LSU (D1/D2 domains) rRNA deposited in NCBI/EMBL (GenBank) under the accession number FN428967.

Deposits: holotype, strain AEG-8-20 (= DSM $26786^{\top}$ ) isolated from grassland soil in Swabian Alb, Baden-Wuerttemberg, Germany $\left(48^{\circ} 25^{\prime} 21.5^{\prime \prime} \mathrm{N}, 9^{\circ} 29^{\prime} 31.6^{\prime \prime} \mathrm{E}\right)$, preserved in a metabolically inactive state at the German Collection of Microorganisms and Cell Cultures, Braunschweig, Germany. Ex-type cultures are deposited at the Centraalbureau voor Schimmelcultures, Utrecht, The Netherlands (CBS $12500^{\top}$ ), the Mycothèque de I'Université Catholique de Louvain (BCCM/MUCL), Louvain-la-Neuve, Belgium (MUCL 54081 ${ }^{\top}$ ) and the Portuguese Yeast Culture Collection (PYCC), Caparica, Portugal (PYCC 6223 ${ }^{\top}$ ).

Strains studied: AEG-8-20, AEG-9-20 (grassland soil, Swabian Alb).

\section{Description of Slooffia velesii Federici,} Röhl \& Begerow, sp. nov. (MB 816165)

Etymology: The specific epithet "velesii" derives from the Slavic mythological figure of Veles. A multifarious god connected with, amongst other things, earth, water and the underworld.

After growth on YM agar plates for 1 mo at $16^{\circ} \mathrm{C}$ and $22^{\circ} \mathrm{C}$, the streak culture is white to off-white, with a smooth and glistening surface. The margin is entire. After growth on YM agar plates for $7 \mathrm{~d}$ at $16^{\circ} \mathrm{C}$, cells are 
subglobose to broadly ellipsoidal (3-6 5-8 $\mu \mathrm{m})$, occurring singly, in pairs or in small clusters, and proliferating by polar budding. Lipid droplets are accumulated in cells with ageing. Pseudohyphae and true hyphae were not observed after $1 \mathrm{mo}$ in Dalmau plate culture on corn meal agar at $16-22{ }^{\circ} \mathrm{C}$.

Assimilation of carbon compounds: growth on D-glucose, D-galactose, L-sorbose, D-xylose, $\mathrm{L}$-arabinose, D-arabinose, sucrose, maltose, a,a-trehalose, cellobiose, lactose, raffinose, melezitose, glycerol, D-glucitol, D-mannitol, myo-inositol, D-glucoronate, DL-lactate, succinate, citrate and ethanol. Weak growth on D-ribose, ribitol, D-saccharic acid, 4hydroxybenzoic acid and 3,4dihydroxybenzoic acid. No growth occurs on D-glucosamine, L-rhamnose, inulin, malic acid, L-tartaric acid, vanillic acid, ferulic acid and trans-ferulic acid. Assimilation of nitrogen compounds: growth on potassium nitrate, sodium nitrite, L-lysine, D-tryptophan and imidazol. Maximum growth temperature: $25^{\circ} \mathrm{C}$.

Molecular characteristics (type strain): nucleotide sequences of LSU (D1/D2 domains) rRNA deposited in NCBI/EMBL (GenBank) under the accession number FN428962.

Deposits: holotype, strain HEG-3-20 (= DSM $\left.102960^{\top}\right)$ isolated from grassland soil in Hainich National Park, Thuringia, Germany $\left(50^{\circ}\right.$ $\left.59^{\prime} 53.1^{\prime \prime} \mathrm{N}, 10^{\circ} 25^{\prime} 58.6^{\prime \prime} \mathrm{E}\right)$, preserved in a metabolically inactive state at the German Collection of Microorganisms and Cell Cultures, Braunschweig, Germany. Ex-type cultures are deposited at the Centraalbureau voor Schimmelcultures, Utrecht, The Netherlands (CBS 11664 $4^{\top}$ ) and the Portuguese Yeast Culture Collection (PYCC), Caparica, Portugal (PYCC 6228).

Strains studied: HEG-3-20 (grassland soil, Hainich National Park), AEG-8-21 (grassland soil, Swabian Alb), HEG-1-20 (grassland soil, Hainich National Park).

\section{Description of Hamamotoa cerberi}

Yurkov, Schäfer \& Begerow, sp. nov. (MB 816164)

Etymology: The specific epithet "cerberi" refers to the three-headed hound Cerberus in Greek and Roman mythology. Cerberus is guarding the gates of the underworld to prevent people escaping from there. Thus, it is living subterraneous like the newly described species.

After growth on YM agar plates for $1 \mathrm{mo}$ at $16^{\circ} \mathrm{C}$ and $22^{\circ} \mathrm{C}$, the streak culture is pale to brownish-cream, butyrous, dull and wrinkled. The margin is entire. After growth on YM agar plates for $7 \mathrm{~d}$ at $16{ }^{\circ} \mathrm{C}$ and $22^{\circ} \mathrm{C}$, cells are ellipsoidal or cylindrical (2-3 8-12 $\mu \mathrm{m})$, occurring singly, in pairs or in small chains. Budding is polar with buds sessile or occurring on short denticles. Ballistospores occasionally present. Pseudohyphae and true hyphae were not observed after 1 mo in Dalmau plate culture on corn meal agar at $16-22^{\circ} \mathrm{C}$.

Assimilation of carbon compounds: growth on D-glucose, D-galactose, D-ribose (delayed), $\mathrm{D}$-xylose, L-arabinose, sucrose, maltose, a, atrehalose, cellobiose, raffinose, melezitose, D-glucitol, D-mannitol, DL-lactate, succinate and citrate. Weak growth on glycerol. No growth occurs on L-sorbose, D-glucosamine, D-arabinose, L-rhamnose (or weak), lactose, inulin, starch, ribitol, myo-inositol, Dglucoronate, ethanol, malic acid, L-tartaric acid, vanillic acid, ferulic acid, trans-ferulic acid, D-saccharic acid, 4-hydroxybenzoic acid and 3,4-dihydroxybenzoic acid. Assimilation of nitrogen compounds: growth on potassium nitrate, sodium nitrite, L-lysine, Dtryptophan and imidazol. Maximum growth temperature: $25^{\circ} \mathrm{C}$.

Molecular characteristics (type strain): nucleotide sequences of LSU (D1/D2 domains) rRNA deposited in NCBI/EMBL (GenBank) under the accession number FN428972. 
Deposits: holotype, strain HEW-1-21 (= DSM $102961^{\top}$ ) isolated from forest soil in Mühlhäuser Stadtwald, Hainich National Park, Thuringia, Germany (51 $11^{\circ} 11^{\prime} 7.3^{\prime \prime} \mathrm{N}, 10^{\circ} 19^{\prime}$ $25.0^{\prime \prime} \mathrm{E}$ ), preserved in a metabolically inactive state at the German Collection of Microorganisms and Cell Cultures, Braunschweig, Germany. Ex-type cultures are deposited at the Centraalbureau voor Schimmelcultures, Utrecht, The Netherlands (CBS 11713 ${ }^{\top}$ ) and the Portuguese Yeast Culture Collection (PYCC), Caparica, Portugal (PYCC 6224 ${ }^{\top}$ ).

Strains studied: HEW-1-21 (forest soil, Hainich National Park).

\section{Description of Hamamotoa telluris \\ Yurkov, Schäfer \& Begerow, sp. nov. (MB 816163)}

Etymology: The specific epithet "telluris" derives from the Roman mythological figure of Tellus, a goddess of the earth.

After growth on YM agar plates for $1 \mathrm{mo}$ at $16{ }^{\circ} \mathrm{C}$ and $22^{\circ} \mathrm{C}$, the streak culture is offwhite to tan, butyrous to mucoid with a smooth and glistening surface. The margin is entire. After growth on YM agar plates for $7 \mathrm{~d}$ at $16{ }^{\circ} \mathrm{C}$ and $22{ }^{\circ} \mathrm{C}$, cells are ellipsoidal or cylindrical (2-3 6-8 $\mu \mathrm{m}$ ), occurring singly or in pairs, and proliferating by polar budding. Pseudohyphae and true hyphae were not observed after $1 \mathrm{mo}$ in Dalmau plate culture on corn meal agar at $16-22{ }^{\circ} \mathrm{C}$.

Assimilation of carbon compounds: growth on D-glucose, L-rhamnose, D-ribose, D-xylose, $\mathrm{D}$-arabinose, sucrose, maltose, a, a-trehalose, lactose, melezitose, glycerol, ribitol, Dglucitol, D-mannitol, myo-inositol, ethanol, Dglucoronate, succinate, citrate, L-tartaric acid, galactaric acid, 4-hydroxybenzoic acid and 3,4-dihydroxybenzoic acid. No growth occurs on D-galactose, L-sorbose, D-glucosamine, L-arabinose, cellobiose, raffinose, inulin, starch, DL-lactate, malic acid, vanillic acid, ferulic acid, trans-ferulic acid and D-saccharic acid. Assimilation of nitrogen compounds: growth on potassium nitrate, sodium nitrite, Llysine, D-tryptophan and imidazol. Maximum growth temperature: $25^{\circ} \mathrm{C}$.

Molecular characteristics (type strain): nucleotide sequences of LSU (D1/D2 domains) rRNA deposited in NCBI/EMBL (GenBank) under the accession number FN428971.

Deposits: holotype, strain HEW-1-23 (= DSM $102968^{\top}$ ) isolated from forest soil in Mühlhäuser Stadtwald, Hainich National Park, Thuringia, Germany (51 $11^{\circ} 7.3^{\prime \prime} \mathrm{N}, 10^{\circ} 19^{\prime}$ $25.0^{\prime \prime} \mathrm{E}$ ), preserved in a metabolically inactive state at the German Collection of Microorganisms and Cell Cultures, Braunschweig, Germany. Ex-type cultures are deposited at the Centraalbureau voor Schimmelcultures, Utrecht, The Netherlands (CBS 11585 ${ }^{\top}$ ) and the Portuguese Yeast Culture Collection (PYCC), Caparica, Portugal (PYCC 6241 ${ }^{\top}$ ).

Strains studied: HEW-1-23 (forest soil, Hainich National Park).

Description of Piskurozyma yama Richter, Mittelbach \& Begerow, sp. nov. (MB 816162)

Etymology: The specific epithet "yama" refers to the god Yama from the Hindu Vedas, a god of death and the underworld. It is said that he was the first mortal who died and now, still seeking for enlightenment, judges the dead.

After one month at $16{ }^{\circ} \mathrm{C}$ and $22^{\circ} \mathrm{C}$ on plates with YM media, the culture is cream to lightly tanned. The texture is butyrous with a dull and smooth surface. Growth is slightly elevated in the center of the colony. Colony margin is wrinkled. After growth on YM agar plates for $7 \mathrm{~d}$ at $16{ }^{\circ} \mathrm{C}$ and $22^{\circ} \mathrm{C}$, cells are ellipsoidal to cylindrical $(3-4 \times 6-10 \mu \mathrm{m})$, occur singly and proliferate by polar budding. Elongated cells in short chains are present. Pseudohyphae and true hyphae were not observed after $1 \mathrm{mo}$ in Dalmau plate culture 
on corn meal agar at $16-22^{\circ} \mathrm{C}$. Chlamydospore-like cells were observed in older ( 3 to 4 weeks) cultures on YM agar incubated at $16^{\circ} \mathrm{C}$. Ballistospores not observed.

Assimilation of carbon compounds: growth on D-glucose, D-galactose, D-xylose, Larabinose, sucrose, maltose, a, a-trehalose, raffinose, melezitose, inulin, starch, ribitol (delayed), D-glucitol, D-mannitol and Dglucuronate. Weak growth on D-arabinose, cellobiose, ethanol and 3,4-dihydroxybenzoic acid. Weak growth reaction in form of a ring occurred on L-sorbose, D-glucosamine, Dribose, L-rhamnose, lactose, glycerol and 4hydroxybenzoic acid. No growth occurs on myo-inositol, DL-lactate, succinate and citrate, D-saccharic acid, malic acid, L-tartaric acid, vanillic acid, ferulic acid and transferulic acid. Assimilation of nitrogen compounds: growth on nitrate, nitrite and Dtryptophan. No growth on L-lysine. Maximum growth temperature: $25^{\circ} \mathrm{C}$.

Molecular characteristics (type strain): nucleotide sequences of ITS-LSU (D1/D2 domains) rRNA deposited in NCBI/EMBL (GenBank) under the accession number HG324302.

Deposits: holotype, strain TSN-538 (= DSM $102965^{\top}$ ) isolated from forest soil in $\mathrm{Na}$ turschutzgebiet Greuthau, Swabian Alb, Baden-Wuerttemberg, Germany ( $48^{\circ} 23^{\prime}$ 56.8" $\mathrm{N}, 9^{\circ} 14^{\prime} 41.4^{\prime \prime} \mathrm{E}$ ), preserved in a metabolically inactive state at the German Collection of Microorganisms and Cell Cultures, Braunschweig, Germany. Ex-type cultures are deposited at the Centraalbureau voor Schimmelcultures, Utrecht, The Netherlands (CBS $12610^{\top}$ ) and the Portuguese Yeast Culture Collection (PYCC), Caparica, Portugal $\left(\right.$ PYCC 6243 ${ }^{\top}$ ).

Strains studied: TSN-538 (forest soil, Swabian Alb).
Description of Piskurozyma tuonelana Lotze-Engelhard, Richter \& Begerow, sp. nov. (MB 816161)

Etymology: The specific epithet "tuonelana" derives from the Finnish mythological figure of Tuonela, a god of the dead and the underworld.

After growth on YM agar plates for 1 mo at $16^{\circ} \mathrm{C}$ and $22^{\circ} \mathrm{C}$, the streak culture is mucous and cream in colour, partially transparent with a glistening surface. Upon ageing, the colony turns dull and tan and appears wrinkled. After growth on YM agar plates for $7 \mathrm{~d}$ at $16{ }^{\circ} \mathrm{C}$ and $22^{\circ} \mathrm{C}$, cells are ellipsoidal to cylindrical (3-4 × 6-10 $\mu \mathrm{m})$, occur singly and proliferate by polar budding. Pseudohyphae and true hyphae were not observed after 1 mo in Dalmau plate culture on corn meal agar at $16-22{ }^{\circ} \mathrm{C}$. Chlamydospore-like cells were observed in older ( 3 to 4 weeks) cultures on YM agar incubated at $16{ }^{\circ} \mathrm{C}$. Ballistospores not observed.

Assimilation of carbon compounds: growth on D-glucose, D-galactose, L-sorbose, D-xylose, L-arabinose, D-arabinose, L-rhamnose, sucrose, maltose, a,a-trehalose, cellobiose, raffinose, melezitose, inulin, ribitol, D-glucitol, D-mannitol, myo-inositol and D-glucoronate. Weak growth on lactose, succinate, citrate, D-saccharid acid, malic acid and L-tartaric acid. No growth occurs on D-glucosamine, Dribose, glycerol, DL-lactate, ethanol, 4hydroxybenzoic acid, 3,4-dihydroxybenzoic acid, vanillic acid, ferulic acid and transferulic acid. Assimilation of nitrogen compounds: nitrate, nitrite, and D-tryptophan. No growth occurs on L-lysine. Maximum growth temperature: $25^{\circ} \mathrm{C}$.

Molecular characteristics (type strain): nucleotide sequences of ITS-LSU (D1/D2 domains) rRNA deposited in NCBI/EMBL (GenBank) under the accession number HG324303. 
Deposits: holotype, strain TSN-649 (= DSM $29565^{\top}$ ) isolated from forest soil in Naturschutzgebiet Höhnriß-Neuben, Swabian Alb, Baden-Wuerttemberg, Germany ( $48^{\circ} 25^{\prime}$ $10.6^{\prime \prime} \mathrm{N}, 9^{\circ} 24^{\prime} 52.9^{\prime \prime} \mathrm{E}$ ), preserved in a metabolically inactive state at the German Collection of Microorganisms and Cell Cultures, Braunschweig, Germany. Ex-type cultures are deposited at the Centraalbureau voor Schimmelcultures, Utrecht, The Netherlands (CBS 12605 ${ }^{\top}$ ) and the Portuguese Yeast Culture Collection (PYCC), Caparica, Portugal (PYCC 6294 ${ }^{\top}$ ).

Strains studied: TSN-649 (forest soil, Swabian Alb).

\section{Description of Dioszegia dumuzii Ebinghaus, Prior \& Begerow, sp. nov. (MB 816160)}

Ethymology: The epithet "dumuzii" derives from the Sumerian mythological figure of Dumuzi, a shepherd god and husband of the goddess Inanna who descended to the underworld where he died later.

After growth on YM agar plates for 1 mo at $16^{\circ} \mathrm{C}$ and $22^{\circ} \mathrm{C}$, the streak culture is reddish-orange, smooth, butyrous, dull and with an entire margin. Young cultures are glistening. Growth is slightly elevated in the centre of the colony. Colony is getting wrinkled after 6 months of incubation. After growth on YM agar plates for 7 days at $16^{\circ} \mathrm{C}$ and $22^{\circ} \mathrm{C}$, cells are ovoidal to ellipsoidal $(3-4 \times 4-6 \mu \mathrm{m})$ but getting progressively spherical upon ageing. Cells occurring singly, in pairs or in small clusters. Ballistospores occasionally present. Pseudohyphae and true hyphae were not observed after 1 mo in Dalmau plate culture on corn meal agar at $16-22{ }^{\circ} \mathrm{C}$. No sexual reproduction was observed.

Assimilation of carbon compounds: growth on D-glucose, D-galactose, D-ribose, D-xylose, L-arabinose, D-arabinose, L-rhamnose, sucrose, maltose, a,a-trehalose, cellobiose, raffinose, melezitose, ribitol, D-mannitol, myo-inositol, D-glucoronate, D-saccharic acid, malic acid, L-tartaric acid, 4hydroxybenzoic acid, 3,4-dihydroxybenzoic acid, vanillic acid, ferulic acid, trans-ferulic acid. Growth reaction in form of a ring occured on ethanol. Weak growth occurs on Dglucosamine, lactose, glycerol, DL-lactate, succinate, citrate. No growth occurs on Lsorbose and inulin. Delayed growth was observed on D-glucitol. Assimilation of nitrogen compounds: nitrite, L-lysine and Dtryptophan. No growth occurs on nitrate. Maximum growth temperature: $28^{\circ} \mathrm{C}$.

Notes: Dioszegia dumuzii has a distinguishable smell. The species is closely related to $D$. cryoxerica, a psychrophilic yeast, which has been isolated from Antarctic. These two species can be distinguished on the basis of maximum growth temperature.

Molecular characteristics (type strain): nucleotide sequences of ITS-LSU (D1/D2 domains) rRNA deposited in NCBI/EMBL (GenBank) under the accession number LT548261.

Deposits: holotype, strain TSN-530 (= DSM $102964^{\top}$ ) isolated from forest soil in $\mathrm{Na}$ turschutzgebiet Greuthau, Swabian Alb, Baden-Wuerttemberg, Germany ( $48^{\circ} 23^{\prime} 56.8^{\prime \prime}$ $\left.\mathrm{N}, 9^{\circ} 14^{\prime} 41.4^{\prime \prime} \mathrm{E}\right)$, preserved in a metabolically inactive state at the German Collection of Microorganisms and Cell Cultures, Braunschweig, Germany. Ex-type cultures are deposited at the Centraalbureau voor Schimmelcultures, Utrecht, The Netherlands (CBS $12501^{\top}$ ) and the Portuguese Yeast Culture Collection (PYCC), Caparica, Portugal $\left(\right.$ PYCC 6248 ${ }^{\top}$ ).

Strains studied: TSN-530, TSN-531 (forest soil, Swabian Alb). 
Description of Chernovia gen. nov. Yurkov \& Begerow (MB 816158)

Etymology: The genus is named in honour of the Russian microbiologist Ivan Yur'evich Chernov for his outstanding contribution to the ecology and systematics of soil yeasts.

The genus is proposed to accommodate the yeast species represented by the strain AY409, which is phylogenetically placed in Wallemiomycetes (Fig. 6) and is distantly related to the genera Basidioascus, Geminibasidium (Nguyen et al. 2013) and Wallemia.

Phylogenetic position: Fungi, Dikarya, Basidiomycota, Wallemiomycetes

Basidiocarps absent. True hyphae and pseudohyphae not observed. Sexual reproduction not observed. Budding cells present. Budding is on the wide basis. Ballistoconidia absent. Arthroconidia absent. Low-weight aromatic compounds can be utilised. Nitrate is utilised.

Type species: Chernovia houtui Federici, Yurkov \& Begerow, sp. nov. (MB 816159)

\section{Description of Chernovia houtui Federici,} Yurkov \& Begerow, sp. nov. (MB 816159)

Ethymology: The epithet "houtui" derives from the Chinese mythological figure of Houtu, a deity of the earth and soil.

After growth on YM agar plates for $7 \mathrm{~d}$ at $16{ }^{\circ} \mathrm{C}$ and $22^{\circ} \mathrm{C}$, the streak culture is cream to yellowish-cream, mucoid with a smooth and glistening surface. The margin is entire. Colony is getting tan and wrinkled after 2 mo of incubation. After growth on YM agar plates for $7 \mathrm{~d}$ at $16{ }^{\circ} \mathrm{C}$ and $22^{\circ} \mathrm{C}$, cells are subglobose to ovoid (3-4 4-7 $\mu \mathrm{m}$ ), occurring singly or in pairs, and proliferating by polar budding. Pseudohyphae and true hyphae were not observed after 1 mo in Dalmau plate culture on corn meal agar at $16-22{ }^{\circ} \mathrm{C}$. Maximum growth temperature: $30^{\circ} \mathrm{C}$.
Assimilation of carbon compounds: growth on D-glucose, D-galactose, D-xylose, Larabinose, sucrose, maltose, a,a-trehalose, cellobiose, raffinose, melezitose, D-glucitol (delayed), D-mannitol, D-glucoronate, DLlactate, succinate, citrate, 4-hydroxybenzoic acid and 3,4-dihydroxybenzoic acid. Growth reaction in form of a ring occurred on ethanol. Weak growth occurs on D-ribose, lactose, glycerol, ribitol, myo-inositol and ferulic acid. No growth occurs on L-sorbose, Dglucosamine, D-arabinose, L-rhamnose, inulin, starch, D-saccharic acid, malic acid, Ltartaric acid, vanillic acid and trans-ferulic acid. Assimilation of nitrogen compounds: nitrate, nitrite, L-lysine and D-tryptophan.

Molecular characteristics (type strain): nucleotide sequences of LSU (D1/D2 domains) rRNA deposited in NCBI/EMBL (GenBank) under the accession number LT548267.

Deposits: holotype, strain AY409 (= DSM $102962^{\top}$ ) isolated from managed pine forest soil in Naturschutzgebiet KienhorstKöllnseen-Eichheide, Schorfheide-Chorin Biosphere Reserve, Brandenburg, Germany (52 $\left.55^{\prime} 14.6^{\prime \prime} \mathrm{N}, 13^{\circ} 38^{\prime} 36.4^{\prime \prime} \mathrm{E}\right)$, preserved in a metabolically inactive state at the German Collection of Microorganisms and Cell Cultures, Braunschweig, Germany. Ex-type cultures are deposited at the Centraalbureau voor Schimmelcultures, Utrecht, The Netherlands (CBS $12316^{\top}$ ), the Mycothèque de I'Université Catholique de Louvain (BCCM/MUCL), Louvain-la-Neuve, Belgium (MUCL 53588 ${ }^{\top}$ ) and the Portuguese Yeast Culture Collection (PYCC), Caparica, Portugal (PYCC 6227 $7^{\top}$ ).

Strains studied: AY409 (forest soil, Schorfheide-Chorin Biosphere Reserve).

\section{Acknowledgements}

Ilse Weßel (Ruhr-University Bochum) is acknowledged for her assistance in the lab during cultivation experiments. Authors are 
grateful to the participants and collaborators of the DFG Biodiversity Exploratories for exchanging and sharing soil samples. We thank Hai D. T. Nguyen for improving the use of English in the manuscript. This work was funded by the DFG (Deutsche Forschungsgemeinschaft) Priority Program 1374 "Infrastructure-Biodiversity-Exploratories", and the research grants from DFG (BE 2201/9-1; BE2201/16-1; YU152/1-1) and DAAD (A/07/94549). Field work permits were issued by the responsible state environmental offices of Baden-Württemberg and Thuringia (according to $\S 72 \mathrm{BbgNatSchG).}$

\section{References}

Blackwell M (2011) The Fungi: 1, 2, 3 ... 5.1 million species? Am J Bot 98:426-438.

Botha A (2011) The importance and ecology of yeasts in soil. Soil Biol Biochem 43:1-8.

Branda E, Turchetti B, Diolaiuti G, Pecci M, Smiraglia $C$, Buzzini $P(2010)$ Yeast and yeast-like diversity in the southernmost glacier of Europe (Calderone Glacier, Apennines, Italy). FEMS Microbiol Ecol 72:354-369.

Connell LB, Redman R, Rodriguez R, Barrett A, Iszard M, Fonseca Á (2010) Dioszegia antarctica sp. nov. and Dioszegia cryoxerica sp. nov., psychrophilic basidiomycetous yeasts from polar desert soils in Antarctica. Int J Syst Evol Microbiol 60:1466-1472.

Connell LB, Redman R, Craig S, Scorzetti G, Iszard M, Rodriguez R (2008) Diversity of soil yeasts isolated from South Victoria Land, Antarctica. Microbial Ecol 56:448-459.

Fell JW (2011) Mrakia Y. Yamada \& Komagata (1987). In: Kurtzman CP, Fell JW \& Boekhout T (eds) The Yeasts, a Taxonomic Study, 5th edn., vol. 3. Elsevier, Amsterdam, pp 1503-1510.

Fierer N, Jackson RB (2006) The diversity and biogeography of soil bacterial communities. $\mathrm{P}$ Natl Acad Sci USA 103:626-631.

Fierer N, Strickland MS, Liptzin D, Bradford MA, Cleveland CC (2009) Global patterns in belowground communities. Ecol Lett 12:1238-1249.
Fischer M, Bossdorf O, Gockel S, et al. (2010) Implementing large-scale and long-term functional biodiversity research: The Biodiversity Exploratories. Basic Appl Ecol 11:473-485.

Fonseca A (1992) Utilization of tartaric acid and related compounds by yeasts: taxonomic implications. Canadian J Microbiol 38:1242-1251.

Glushakova AM, Maximova IA, Kachalkin AV, Yurkov AM (2010) Ogataea cecidiorum sp. nov., a methanol-assimilating yeast isolated from galls on willow leaves. Anton Leeuw Int J G 98:93-101.

Golubtsova YV, Glushakova AM, Chernov IY (2007) The seasonal dynamics of yeast communities in the rhizosphere of soddy-podzolic soils. Eurasian Soil Sci+ 40:875-879.

Hawksworth DL (2001) The magnitude of fungal diversity: the 1.5 million species estimate revisited. Mycol Res 105:1422-1432.

Jensen V (1963) Studies on the microflora of Danish beech forest soils. IV. Yeasts and yeast-like fungi. Zbl Bakt II Natur 117:41-65.

Katoh K, Standley DM (2013) MAFFT multiple sequence alignment software version 7: improvements in performance and usability. Mol Biol Evol 30:772-780.

Kurtzman CP, Fell JW, Boekhout T, Robert V (2011) Methods for isolation, phenotypic characterization and maintenance of yeasts. In: Kurtzman CP, Fell JW \& Boekhout T (eds) The Yeasts, a Taxonomic Study, 5th edn., vol. 1. Elsevier, Amsterdam, pp 87-111.

Liu XZ, Wang QM, Göker M, et al. (2015) Towards an integrated phylogenetic classification of the Tremellomycetes. Stud Mycol 81:85-147.

Lynch MD, Neufeld JD (2015) Ecology and exploration of the rare biosphere. Nat Rev Microbiol 13:217-229.

Maksimova IA, Chernov IY (2004) Community structure of yeast fungi in forest biogeocenoses. Microbiology 73:474-481.

Nasr S, Soudi MR, Nasrabadi SMZ, Nikou MM, Salmanian AH, Nguyen HD (2014) Basidioascus persicus sp. nov., a yeast-like species of the order Geminibasidiales isolated from soil. Int J Syst Evol Microbiol 64:3046-3052. 
Nguyen HD, Nickerson NL, Seifert KA (2013) Basidioascus and Geminibasidium: a new lineage of heat-resistant and xerotolerant basidiomycetes. Mycologia 105:1231-1250.

Sampaio JP (1999) Utilization of low molecular weight aromatic compounds by heterobasidiomycetous yeasts: taxonomic implications. Canadian J Microbiol 45:491-512.

Sampaio JP, Gadanho M, Bauer R, Weiß M (2003) Taxonomic studies in the Microbotryomycetidae: Leucosporidium golubevii sp. nov., Leucosporidiella gen. nov. and the new orders Leucosporidiales and Sporidiobolales. Mycol Prog 2:5368.

Sampaio JP, Gadanho M, Santos S, Duarte FL, Pais C, Fonseca A, Fell JW (2001) Polyphasic taxonomy of the basidiomycetous yeast genus Rhodosporidium: Rhodosporidium kratochvilovae and related anamorphic species. Int J Syst Evol Microbiol 51:687-697.

Silvestro D, Michalak I (2012) raxmIGUI: a graphical front-end for RAxML. Org Divers Evol 12:335337.

Sogin ML, Morrison HG, Huber JA, et al. (2006) Microbial diversity in the deep sea and the underexplored "rare biosphere". P Natl Acad Sci USA 103:12115-12120.

Stamatakis A, Hoover P, Rougemont J (2008) A rapid bootstrap algorithm for the RAxML web servers. Syst Biol 57:758-771.

Taylor DL, Hollingsworth TN, McFarland JW, Lennon NJ, Nusbaum C, Ruess RW (2014) A first comprehensive census of fungi in soil reveals both hyperdiversity and fine-scale niche partitioning. Ecol Monogr 84:3-20.

Tedersoo L, Bahram M, Põlme S, et al. (2014) Global diversity and geography of soil fungi. Science 346:1256688.

Vishniac HS (2006) A multivariate analysis of soil yeasts isolated from a latitudinal gradient. Microbial Ecol 52:90-103.

Wang QM, Yurkov AM, Göker M, et al. (2015) Phylogenetic classification of yeasts and related taxa within Pucciniomycotina. Stud Mycol 81:149189.

Wubet T, Christ S, Schöning I, et al. (2012) Differences in soil fungal communities between Euro- pean beech (Fagus sylvatica L.) dominated forests are related to soil and understory vegetation. PloS One 7:e47500.

Yarrow, D. (1998) Methods for the isolation, maintenance and identification of yeasts. In: Kurtzman CP, Fell JW (eds) The yeasts: a taxonomic study, 4th edn. Elsevier, Amsterdam, pp 77-100.

Yurkov AM, Schäfer AM, Begerow D (2009a) Clavispora reshetovae. Fungal Planet 35. Persoonia 23:182-183.

Yurkov AM, Schäfer AM, Begerow D (2009b) Barnettozyma vustinii. Fungal Planet 38. Persoonia 23:188-189.

Yurkov AM, Kemler M, Begerow D (2011) Species accumulation curves and incidence-based species richness estimators to appraise the diversity of cultivable yeasts from beech forest soils. PLoS One 6:e23671.

Yurkov AM, Kemler M, Begerow D (2012a) Assessment of yeast diversity in soils under different management regimes. Fungal Ecol 5:24-35.

Yurkov AM, Schäfer AM, Begerow D (2012b). Leucosporidium drummii sp. nov., a member of the Microbotryomycetes isolated from soil. Int $\mathrm{J}$ Syst Evol Microbiol 62:728-734.

Yurkov A, Wehde T, Kahl T, Begerow D (2012c) Aboveground deadwood deposition supports development of soil yeasts. Diversity (Basel) 4:453474.

Yurkov A, Inácio J, Chernov IY, Fonseca Á (2015) Yeast biogeography and the effects of species recognition approaches: the case study of widespread basidiomycetous species from birch forests in Russia. Curr Microbiol 70:587-601.

Yurkov AM, Röhl O, Pontes A, Carvalho C, Maldonado C, Sampaio JP (2016) Local climatic conditions constrain soil yeast diversity patterns in Mediterranean forests, woodlands and scrub biome. FEMS Yeast Res 16: fov103. 
Table 1 - Yeast species isolated from forest soils

\begin{tabular}{|c|c|c|c|c|c|}
\hline Species & Apr-2008 & Apr-2009 & Jun-2009 & Aug-2009 & Oct-2009 \\
\hline \multicolumn{6}{|l|}{ Ascomycetes } \\
\hline Arthroascus schoenii & $X^{* \star}$ & & & & \\
\hline Aureobasidium pullulans & & & & $x$ & \\
\hline Barnettozyma pratensis & $\mathrm{X}$ & $x$ & $x$ & & $x$ \\
\hline Barnettozyma vustinii & $\mathrm{X}$ & & & & \\
\hline Candida kruisii & $X$ & $x$ & $x$ & $x$ & $x$ \\
\hline Candida sake & $\mathrm{X}^{*}$ & $x$ & $x$ & $x$ & \\
\hline Candida santamariae & & $x$ & $x$ & $x$ & \\
\hline Candida sp. (TSN-747, 806) & & $x$ & & $x$ & \\
\hline Candida vartiovaarae & $\mathrm{X} / \mathrm{X}^{*}$ & & & & \\
\hline Cyberlindnera misumaiensis & $\mathrm{X}$ & & & $x$ & \\
\hline Debaryomyces hansenii & $\mathrm{X} / \mathrm{X}^{*}$ & & & & \\
\hline Kazachstania piceae & $\mathrm{X} / \mathrm{X}^{*}$ & $x$ & $x$ & $x$ & $x$ \\
\hline “Saccharomyces” sp. (TSN-505) & & & $x$ & & \\
\hline Schwanniomyces capriottii & $\mathrm{X}$ & & $x$ & & \\
\hline \multicolumn{6}{|l|}{ Basidiomycetes } \\
\hline Apiotrichum dulcitum & $\mathrm{X} / \mathrm{X}^{*}$ & $x$ & $x$ & $x$ & $x$ \\
\hline Apiotrichum porosum & $\mathrm{X} / \mathrm{X}^{*}$ & $x$ & & $x$ & $x$ \\
\hline Chernovia houtui gen. nov. sp. nov. & $\mathrm{X}^{* *}$ & & & & \\
\hline Colacogloea demeterae sp. nov. & $\mathrm{X} / \mathrm{X}^{*}$ & & & & \\
\hline Curvibasidium cygneicollum & & & & $x$ & $x$ \\
\hline Cutaneotrichosporon moniliiforme & & $x$ & $x$ & $x$ & \\
\hline Cystofilobasdium capitatum & & $x$ & & $x$ & $x$ \\
\hline Cystofilobasdium macerans & $\mathrm{X}$ & & & & \\
\hline Dioszegia dumuzii sp. nov. & & & & & $x$ \\
\hline Filobasidium stepposum & & & & & $x$ \\
\hline Filobasidium wieringae & & & & & $x$ \\
\hline Goffeauzyma gastrica & $\mathrm{X}$ & $x$ & $x$ & $x$ & $x$ \\
\hline Hamamotoa cerberi sp. nov. & $\mathrm{X}^{*}$ & & & & \\
\hline Hamamotoa telluris sp. nov. & $\mathrm{X}^{*}$ & & & & \\
\hline Holtermanniella takashimae & & $x$ & $x$ & & \\
\hline Holtermanniella wattica & $\mathrm{X} / \mathrm{X}^{*}$ & & & & \\
\hline Leucosporidium scottii & & & $x$ & $x$ & $x$ \\
\hline Mrakia frigida & & & & & $x$ \\
\hline Mrakia gelida & & & & $x$ & \\
\hline Naganishia adeliensis & & & & $x$ & \\
\hline Piskurozyma filicatus & & & $x$ & $x$ & \\
\hline Piskurozyma tuonelana sp. nov. & & & & $x$ & \\
\hline Piskurozyma yama sp. nov. & & & & $x$ & \\
\hline Rhodotorula colostri & & & $x$ & & $x$ \\
\hline “Rhodotorula” sp. CBS 11560 & $\mathrm{X}^{*}$ & & & & \\
\hline “Rhodotorula” sp. (TSN-574) & & $x$ & & & \\
\hline Saitozyma podzolica & $\mathrm{X}$ & $x$ & $x$ & $x$ & $x$ \\
\hline
\end{tabular}




\begin{tabular}{|l|l|l|l|l|l|}
\hline Slooffia velesii sp. nov. & $\mathrm{X}^{*}$ & & & & \\
\hline Solicoccozyma aeria & & $\mathrm{X}$ & $\mathrm{X}$ & $\mathrm{X}$ & $\mathrm{X}$ \\
\hline Solicoccozyma terrea & $\mathrm{X}$ & $\mathrm{X}$ & $\mathrm{X}$ & $\mathrm{X}$ & \\
\hline Solicoccozyma terricola & $\mathrm{X}$ & $\mathrm{X}$ & $\mathrm{X}$ & $\mathrm{X}$ & $\mathrm{X}$ \\
\hline Tausonia pullulans & $\mathrm{X} / \mathrm{X}^{*}$ & $\mathrm{X}$ & $\mathrm{X}$ & $\mathrm{X}$ & $\mathrm{X}$ \\
\hline Vanrija albida & $\mathrm{X}$ & & & & \\
\hline Vishniacozyma victoriae & & $\mathrm{X}$ & & & $\mathrm{X}$ \\
\hline Vishniacozyma sp. CBS 12229 & $\mathrm{X}^{* *}$ & & & & \\
\hline
\end{tabular}

Note: $X$ - isolated on nutrient-rich medium; $X^{*}$ - isolated on a nitrogen-depleted medium; $X^{* *}-$ single strains isolated by Yurkov et al. (2012a) and re-identified in the present study.

Table 2 - Number of yeast species isolated from studied forest plots

\begin{tabular}{|l|c|c|c|c|c|}
\hline $\begin{array}{l}\text { Region of isolation, forest } \\
\text { management }\end{array}$ & Apr-2008 * & Apr-2009 & Jun-2009 & Aug-2009 & Oct-2009 \\
\hline Hainich, near-natural & 9 & 5 & 6 & 8 & 8 \\
\hline Hainich, managed & 14 & 13 & 11 & 7 & 9 \\
\hline Swabian Alb, near-natural & 8 & 8 & 8 & 11 & 8 \\
\hline Swabian Alb, managed & 12 & 9 & 9 & 8 & 8 \\
\hline
\end{tabular}

Note: * - includes species reported by Yurkov et al. (2012a) and species isolated on nitrogen-depleted media reported in this manuscript. 


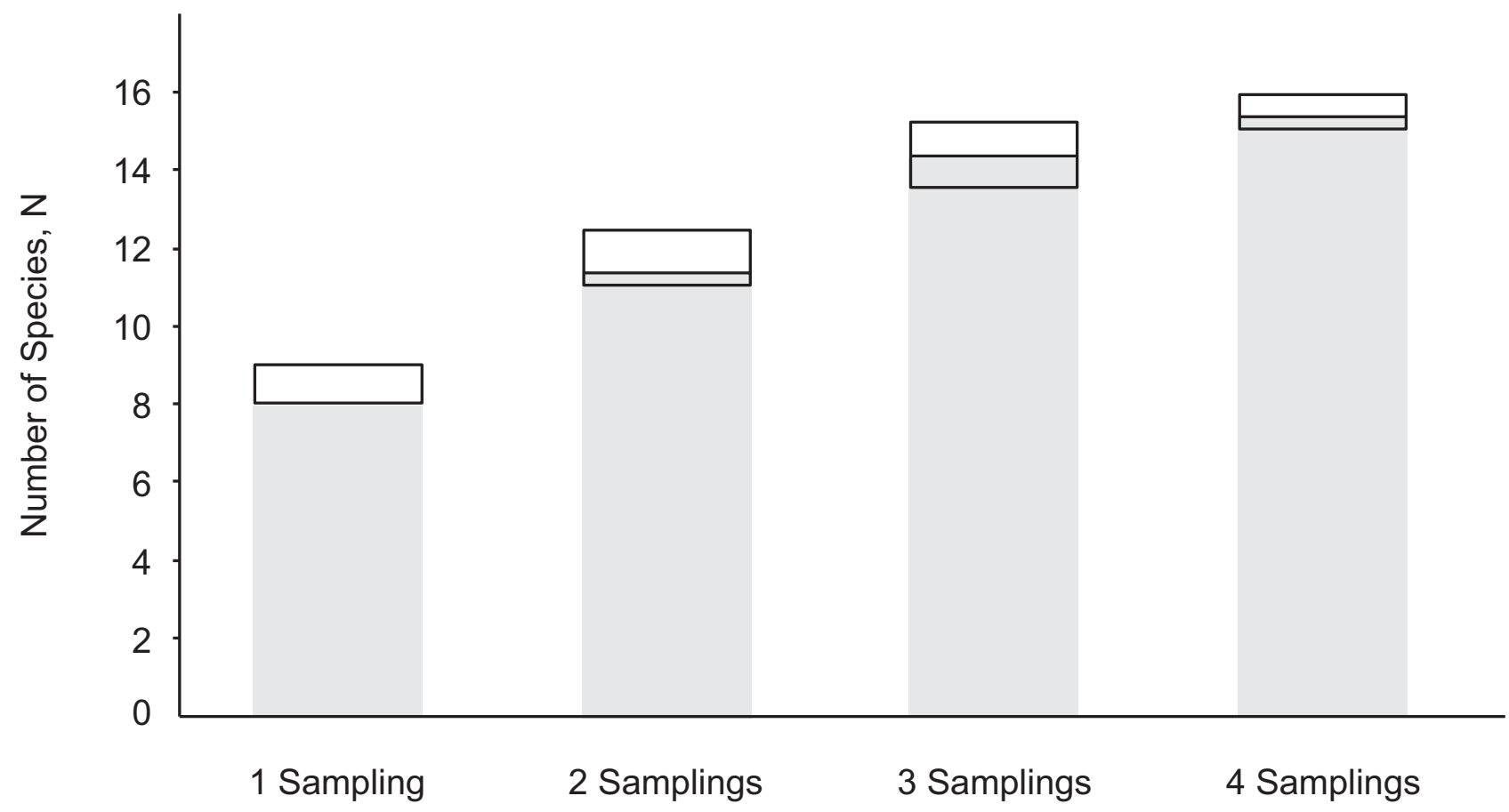

Figure 1 - Box plots showing species richness values (25\% quartile, median, $75 \%$ quartile) observed in beech forest soils after randomised 1, 2, 3 and 4 samplings.

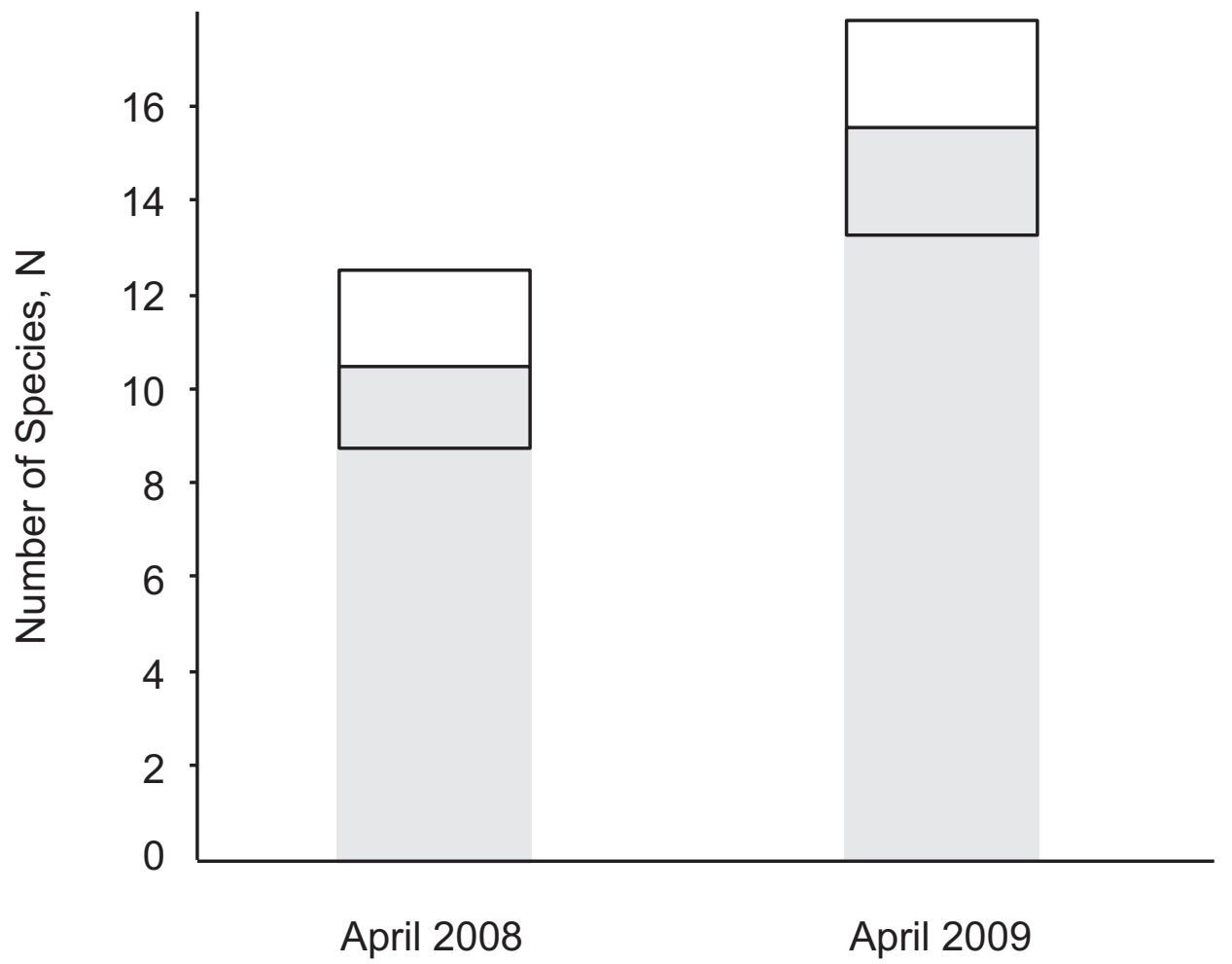

Figure 2 - Box plots showing species richness values (25\% quartile, median, $75 \%$ quartile) observed in beech forest soils sampled in April 2008 and 2009. 


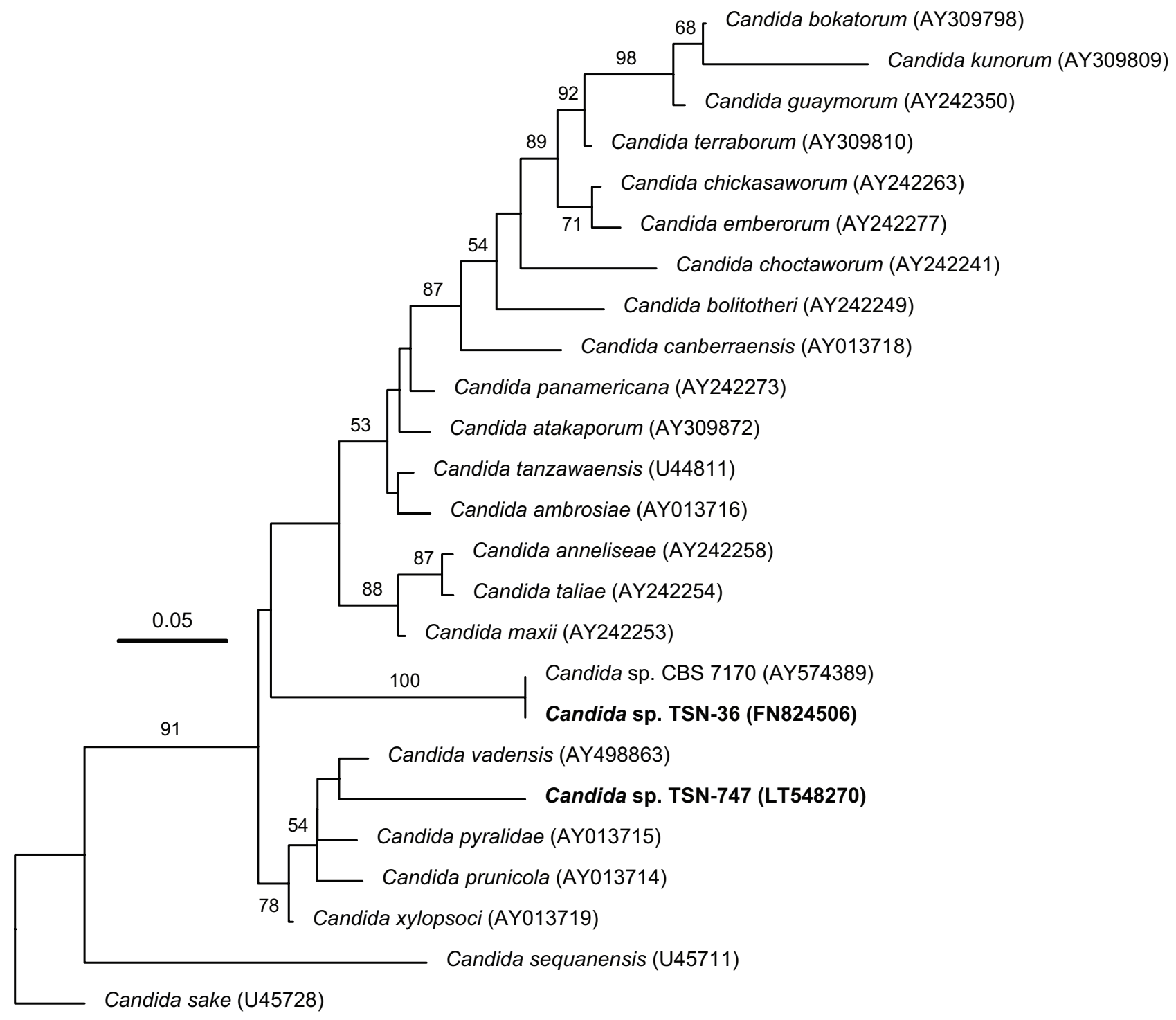

Figure 3 - Maximum likelihood analysis of an alignment of the LSU rRNA gene (D1/D2 domains) for the Candida tanzawaensis clade. The numbers provided on branches are frequencies (>50\%) with which a given branch appeared in 100 bootstrap replications. The scale bars indicate the numbers of expected substitutions accumulated per site. 


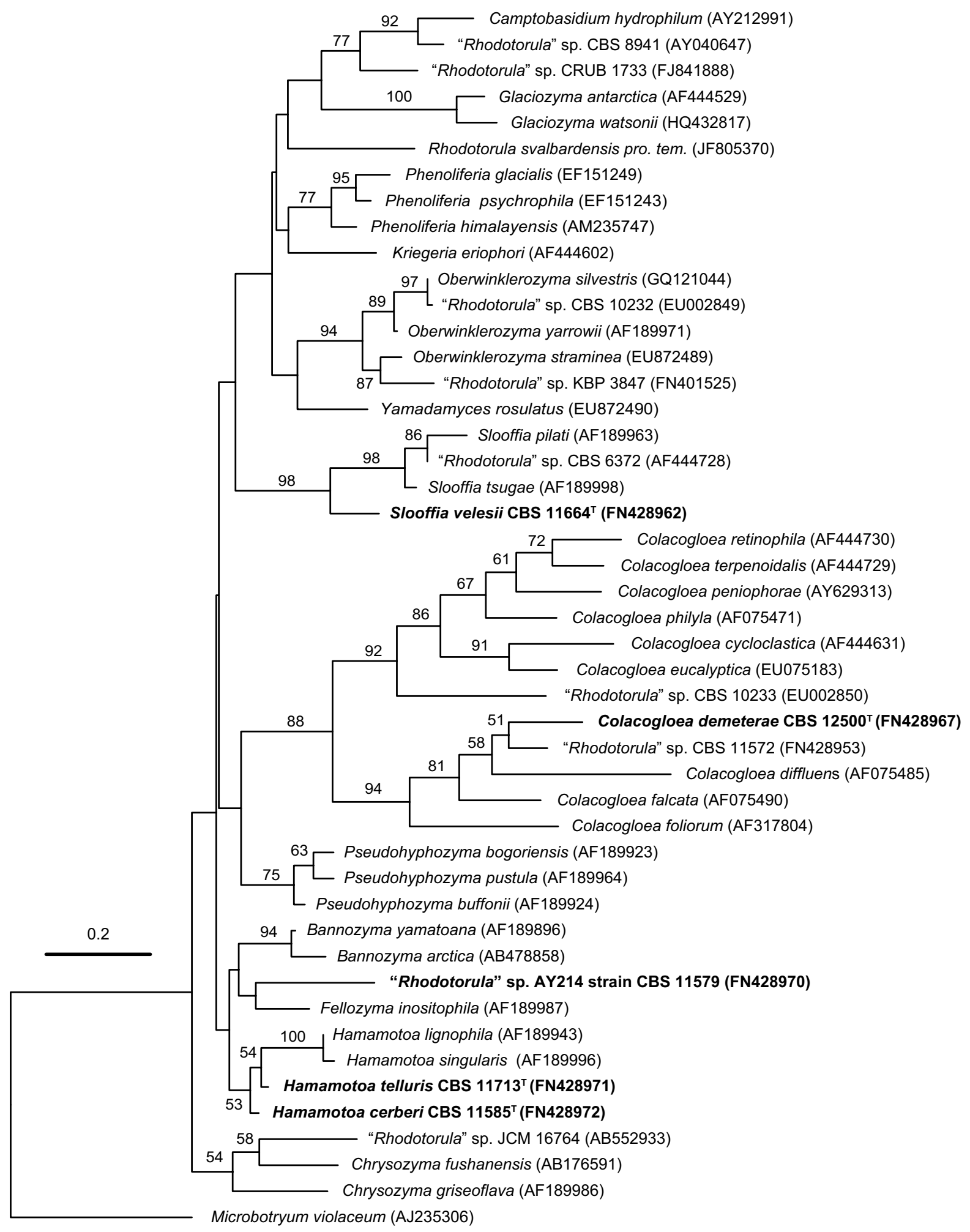

Figure 4 - Phylogenetic relationships of yeasts and related taxa from the Microbotryomycetes lineage obtained by Neighbor-Joining analysis of LSU (D1/D2 domains) rRNA using the Kimura 2-Parameter method and the rate variation among sites was modelled with a gamma distribution (shape parameter $=0.38$ ) as implemented in MEGA7 software. The numbers provided on branches are frequencies ( $>50 \%$ ) with which a given branch appeared in 100 bootstrap replications. The scale bars indicate the numbers of expected substitutions accumulated per site. 


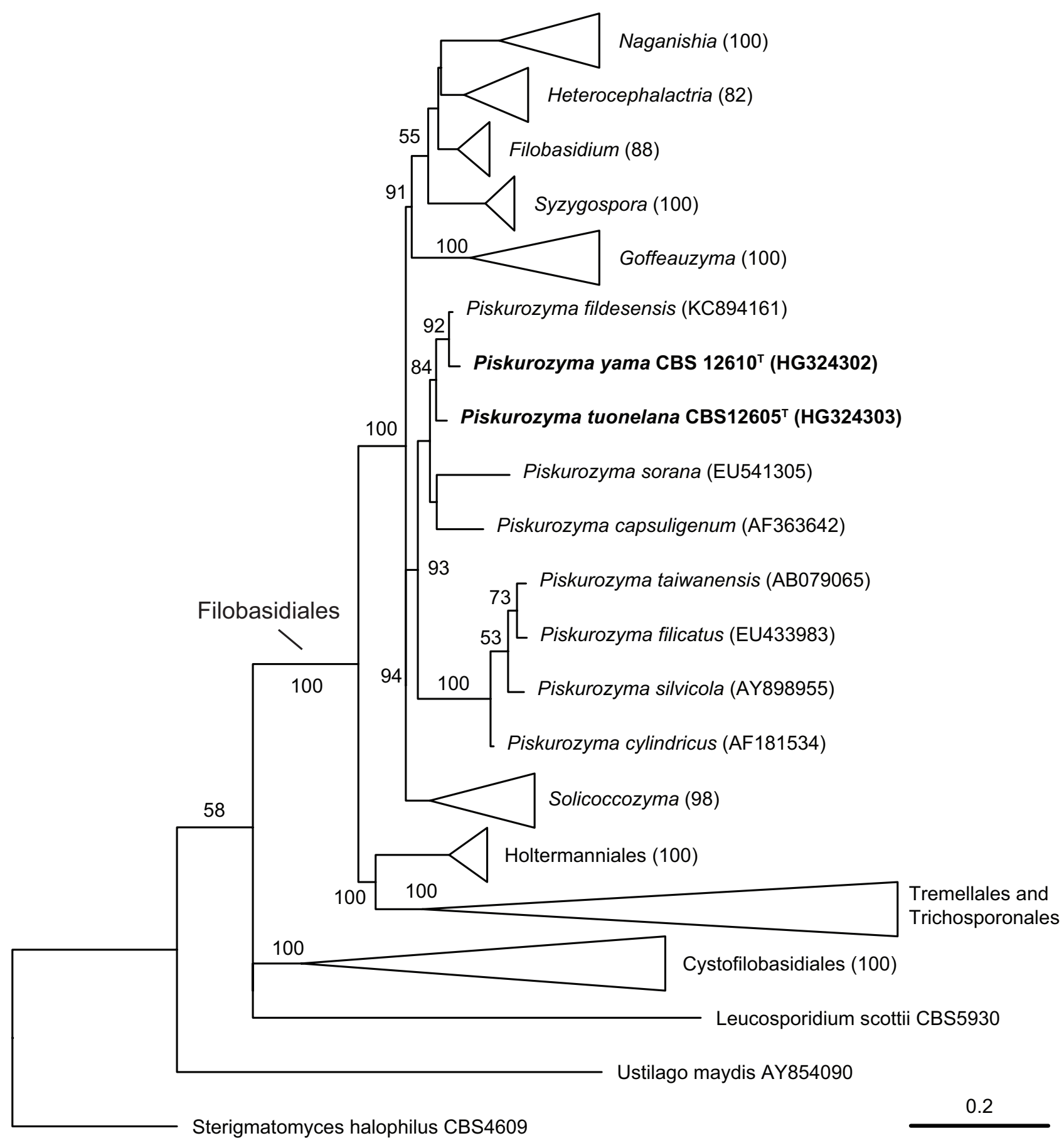

Figure 5 - Maximum likelihood analysis of an alignment of the LSU rRNA gene (D1/D2 domains) for the genus Piskurozyma. Branches corresponding to clades other than Piskurozyma are collapsed. The numbers provided on branches are frequencies $(>50 \%)$ with which a given branch appeared in 100 bootstrap replications. The scale bars indicate the numbers of expected substitutions accumulated per site. 


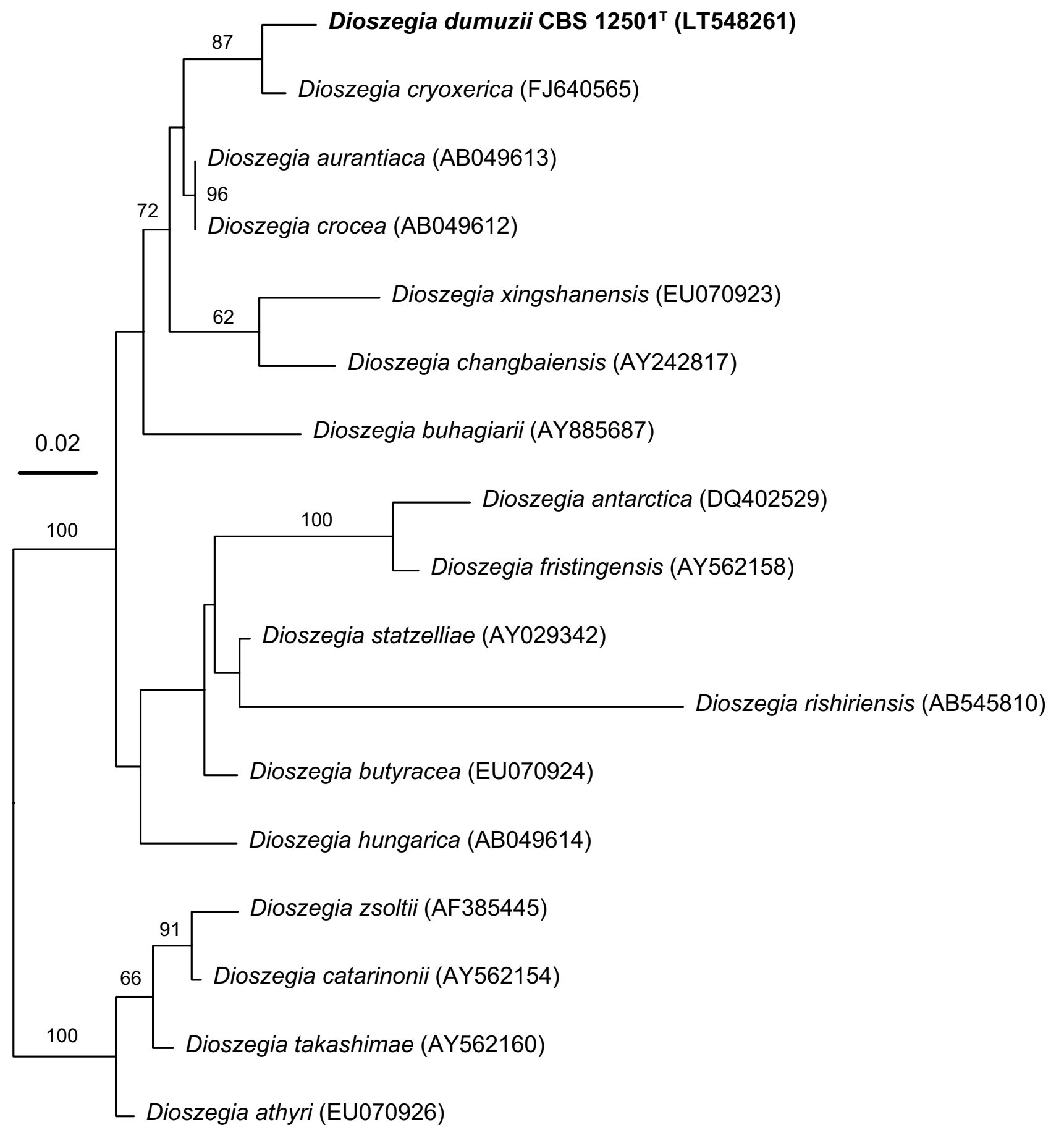

Figure 6 - Unrooted tree illustrating phylogenetic placement of Dioszegia dumuzii obtained by maximum likelihood analysis of the ITS region. The numbers provided on branches are frequencies ( $>50 \%$ ) with which a given branch appeared in 100 bootstrap replications. The scale bars indicate the numbers of expected substitutions accumulated per site. 


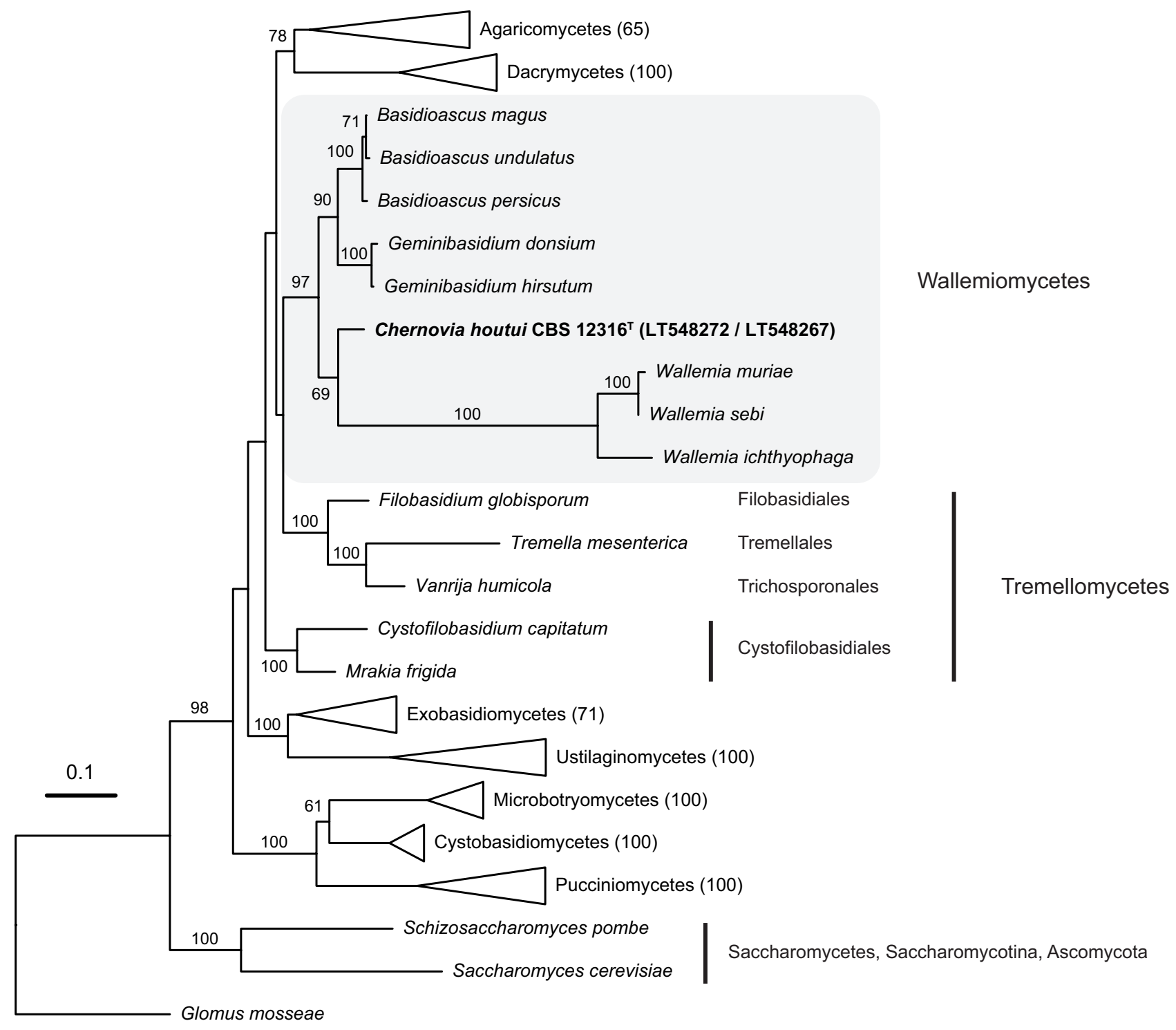

Figure 7 - Maximum likelihood analysis of an alignment of the SSU rRNA and the LSU (D1/D2 domains) rRNA showing phylogenetic relationships of Wallemiomycetes and other families comprising the phylum Basidiomycota. The analysis is based on the dataset TreeBase S13396 (Nguyen et al. 2013). Branches corresponding to classes other than Wallemiomycetes and Tremellomycetes are collapsed. The numbers provided on branches are frequencies $(>50 \%$ ) with which a given branch appeared in 100 bootstrap replications. The scale bars indicate the numbers of expected substitutions accumulated per site. 

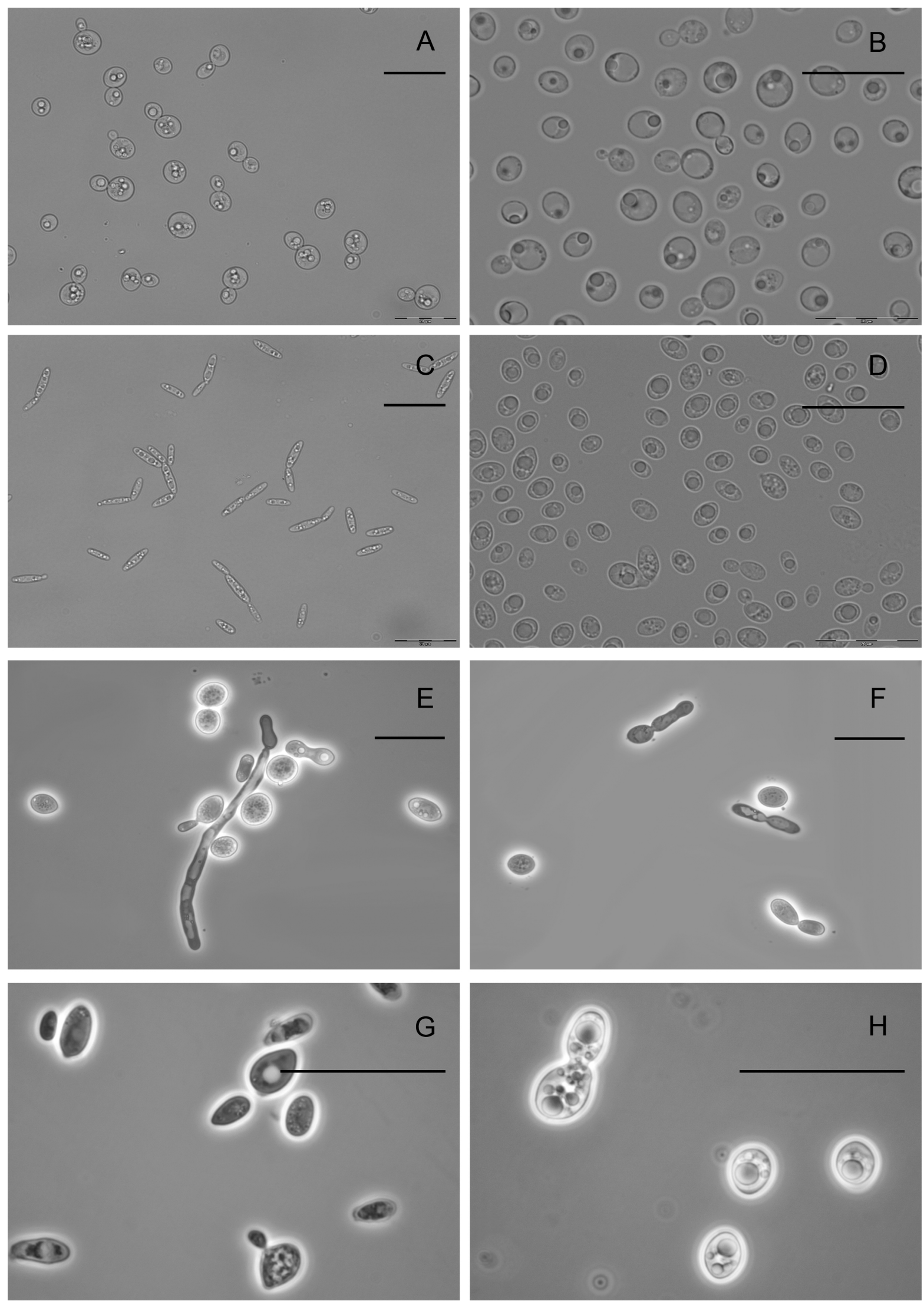

Figure 8 - Phase contrast micrographs illustrating cell morphology of Colacogloea demeterae (A), Slooffia velesii (B), Hamamotoa cerberi (C), Hamamotoa telluris (D), Piskurozyma yama (E), Piskurozyma tuonelana (F), Dioszegia dumuzii (G) and Chernovia houtui (H). Bar = $20 \mu \mathrm{m}$. 


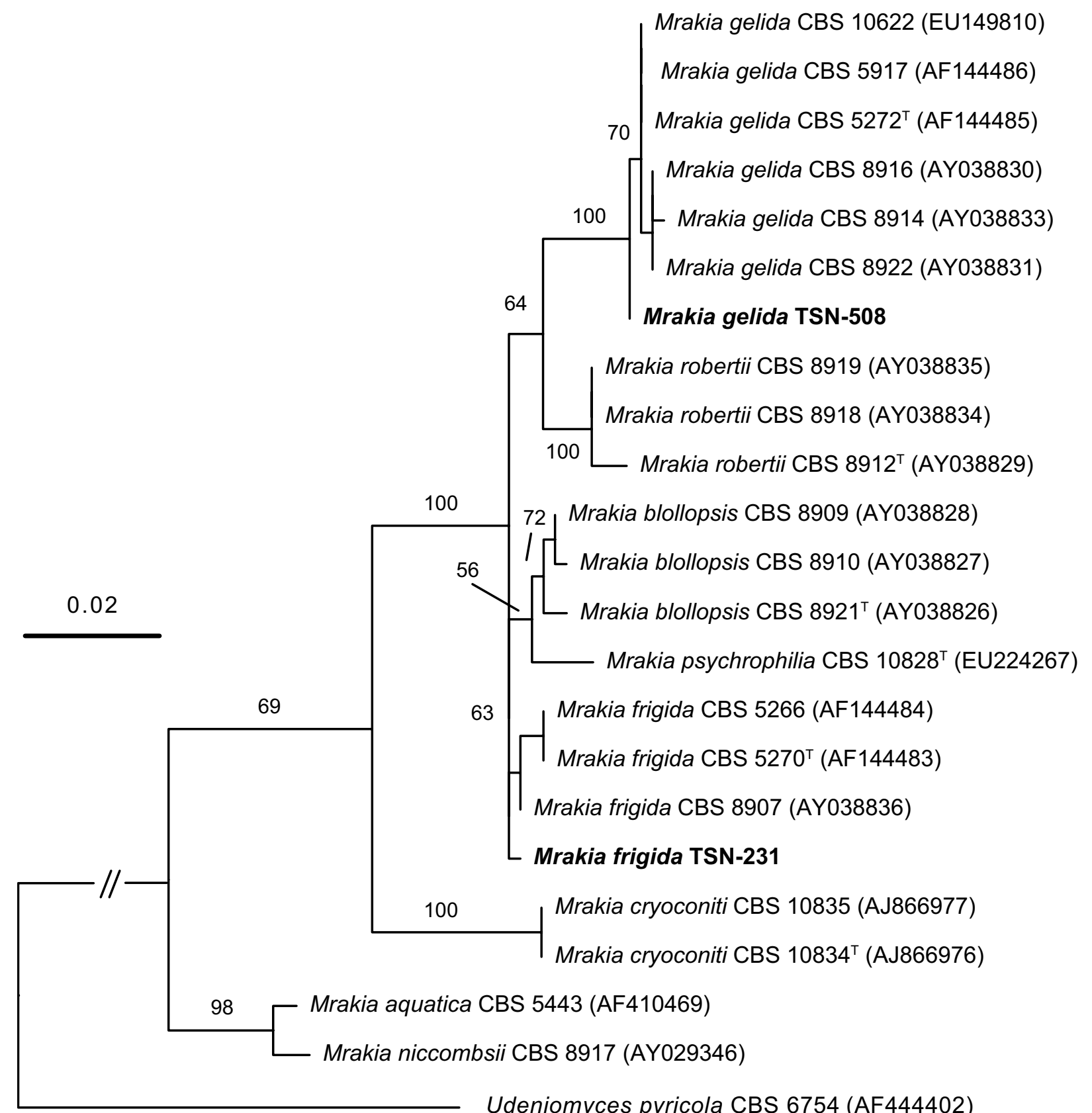

Figure S1 - Maximum likelihood analysis of an alignment of the ITS region for the genus Mrakia. The numbers provided on branches are frequencies (>50\%) with which a given branch appeared in 100 bootstrap replications. The scale bars indicate the numbers of expected substitutions accumulated per site. Type strains are designated with the symbol ${ }^{\top}$. 